\title{
Molecular dynamics simulations of elementary chemical processes in liquid water using combined density functional and molecular mechanics potentials. II. Charge separation processes
}

\author{
M. Strnad, ${ }^{\text {a) }}$ M. T. C. Martins-Costa, and C. Millot \\ Laboratoire de Chimie Théorique, URA CNRS 510, Institut Nancéien de Chimie Moléculaire, \\ Université Henri Poincaré-Nancy I, BP 239, 54506 Vandoeuvre-lès-Nancy Cedex, France \\ I. Tuñón \\ Laboratoire de Chimie Théorique, URA CNRS 510, Institut Nancéien de Chimie Moléculaire, \\ Université Henri Poincaré-Nancy I, BP 239, 54506 Vandoeuvre-lès-Nancy Cedex-France and Departamento \\ de Quimica Física Universidad de Valencia, 46100 Burjasot, Spain \\ M. F. Ruiz-López and J. L. Rivail \\ Laboratoire de Chimie Théorique, URA CNRS 510, Institut Nancéien de Chimie Moléculaire, \\ Université Henri Poincaré-Nancy I, BP 239, 54506 Vandoeuvre-lès-Nancy Cedex, France
}

(Received 23 September 1996; accepted 25 November 1996)

\begin{abstract}
A new approach to carry out molecular dynamics simulations of chemical reactions in solution using combined density functional theory/molecular mechanics potentials is presented. We focus our attention on the analysis of reactive trajectories, dynamic solvent effects and transmission coefficient rather than on the evaluation of free energy which is another important topic that will be examined elsewhere. In a previous paper we have described the generalities of this hybrid molecular dynamics method and it has been employed to investigate low energy barrier proton transfer process in water. The study of processes with activation energies larger than a few $k T$ requires the use of specific techniques adapted to "rare events" simulations. We describe here a method that consists in the simulation of short trajectories starting from an equilibrated transition state in solution, the structure of which has been approximately established. This calculation is particularly efficient when carried out with parallel computers since the study of a reactive process is decomposed in a set of short time trajectories that are completely independent. The procedure is close to that used by other authors in the context of classical molecular dynamics but present the advantage of describing the chemical system with rigorous quantum mechanical calculations. It is illustrated through the study of the first reaction step in electrophilic bromination of ethylene in water. This elementary process is representative of many charge separation reactions for which static and dynamic solvent effects play a fundamental role. (C) 1997 American Institute of Physics. [S0021-9606(97)00809-X]
\end{abstract}

\section{INTRODUCTION}

Applications of molecular dynamics (MD) simulations to the study of chemical reactions in solution have been recently reviewed by Whitnell and Wilson. ${ }^{1}$ So far, most of the works have been devoted to the study of fundamental processes such as ion pair association, ${ }^{2} S_{N} 1^{3}$ and $S_{N} 2^{4}$ reactions and electron ${ }^{5}$ and proton transfer. ${ }^{6}$ The main interest for carrying out MD simulations, for instance with respect to calculations using simple solvent models such as the selfconsistent reaction field (SCRF) method, ${ }^{7}$ lies in the fact that solvent dynamics may play an important role on the reaction mechanism and energetics. Whereas most SCRF approaches are based on solute-solvent equilibrium hypothesis and neglect dynamic solvent effects (see, however, Ref. 8), nonequilibrium solvation can be naturally accounted for in molecular dynamics. The main limitation of classical MD simulations to study reactions in solution lies in the use of

\footnotetext{
${ }^{a)}$ Permanent address: E. Hála Laboratory of Thermodynamics, Institute of Chemical Process Fundamentals, Academy of Sciences, 16502 Prague 6-Suchdol, Czech Republic.
}

classical potentials. While this may be sufficient to represent the solvent, the chemical system cannot be described accurately with such potentials since bonds are being formed and broken and electronic effects are crucial. This shortcoming is overcome with the use of hybrid quantum mechanics/ molecular mechanics $(\mathrm{QM} / \mathrm{MM})$ potentials that have known a growing interest in the last years. ${ }^{9}$

In a preceding paper, ${ }^{10}$ we have reported the first application of hybrid QM/MM molecular dynamics to the simulation of a chemical reaction in solution at the ab initio level. More precisely, we have used a density functional theory/ molecular mechanics approach (DFT/MM) recently implemented. ${ }^{11,12}$ In such a work, ${ }^{10}$ we have studied the dynamics of proton transfer in a strongly hydrogen-bonded system in liquid water, namely, the hydroxyl-water complex. The transfer process has a low activation barrier and many reactive events can be observed in a few picosecond simulation; typically the proton jumps from water to hydroxyl each picosecond. However, most chemical processes have larger activation energies and the probability for a reactive event to occur may be too small for a process to be observed in a 


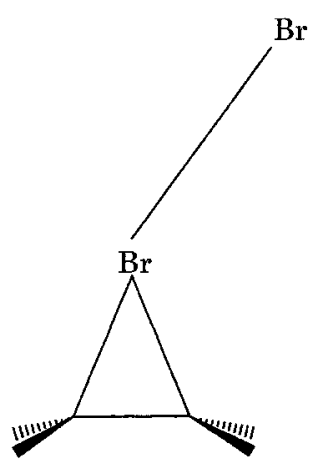

TS $\mathbf{C}_{\mathrm{s}}$

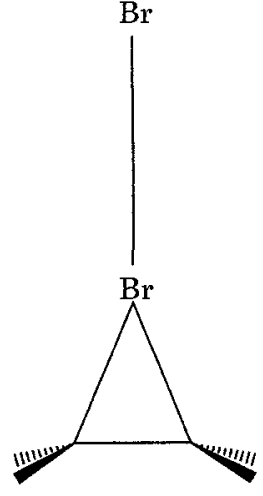

TS $\mathbf{C}_{2 v}$
FIG. 1. Schematic structure of the transition state with $C_{s}$ (left) or $C_{2 v}$ symmetry.

limited simulation time at usual temperatures. Alternatively, one may use some ad hoc MD techniques such as those developed to simulate rare events. ${ }^{13-14}$ The aim of this work is precisely to implement a rare event technique within the context of hybrid DFT/MM MD simulations and to analyse the dynamics of a charge separation process in water and to compute the transmission coefficient for that reaction. ${ }^{15}$

Let us comment further on the role of solvent on reaction dynamics. The validity of transition state theory was questioned by Kramers ${ }^{16}$ in the case of reactions in solution. The underlying idea is that for many processes in polar media, the solvent cannot follow the chemical system so that nonequilibrium solvation is foreseen. There are different perspectives from which interpretation of non-equilibrium effects on reactions can be done. To summarize, two principal mechanisms may be considered. In the first one, the solvent is in equilibrium with the chemical system until a given point in the reaction coordinate. Then, the system crosses the barrier with an essentially solvent frozen configuration. In that case, the main effect is a net increase of the activation barrier and therefore a decrease of the reaction rate. In the second mechanism, one assumes that the solute and solvent are in equilibrium at the top of the barrier and this minimizes the activation energy. If the solvent cannot be equilibrated with the chemical system all along the reaction coordinate this implies that the climb to the transition barrier must be preceded by a convenient fluctuation of the solvent suitable to solvate the transition state. The last mechanism has been in general assumed in MD studies of reactions in solution having large activation energies.

In this work, we shall study a basic reaction in organic chemistry: ethylene bromination in water. The mechanism for this process was postulated in the thirties ${ }^{17}$ and is generally assumed to be a two-step trans-addition involving the bromonium cation as the key intermediate. We shall focus our attention on the first rate-limiting step of the process which is schematized in Scheme 1 and is essentially a charge separation process $\mathbf{A B} \rightarrow \mathbf{A}^{+}+\mathbf{B}^{-}$. Ethylene and bromine first form a charge transfer complex (CTC) that is expected to

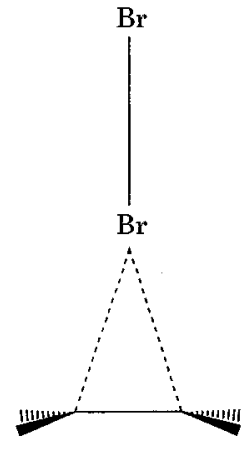

have a small or moderate polarity. The CTC then dissociates to give the bromonium cation and a bromide anion. This charge separation process is extremely sensitive to the solvent nature as shown by the relative value of the bromination rate constant $k^{\mathrm{S}} / k^{\mathrm{CCl} 4}$ which is $1.610^{5}$ for $\mathrm{S}=\mathrm{CH}_{3} \mathrm{OH}$ and $1.110^{10}$ for $\mathrm{S}=$ water. $^{18}$ The role of the solvent in bromination has been extensively studied. ${ }^{19} \mathrm{~A}$ recent review on this reaction has been reported by Ruasse ${ }^{20}$ including a detailed discussion on open questions such as the nature of bromonium ions (cyclic, open) or the reversibility of the bromonium ion formation.

The reaction of halogens with ethylenic compounds has been theoretically studied in the gas phase by a few authors. ${ }^{20-27}$ Clearly, the process in Scheme 1 is not possible but a transition state with Cs symmetry (see Fig. 1) has been reported. ${ }^{23}$ Some results in solution have also been reported. ${ }^{25-27} \mathrm{Ab}$ initio calculations using a cavity model to represent the solvent showed that the symmetry of the transition state remains $C_{S}$ in non-polar solvents but changes to the classical $C_{2 v}$ structure (see Fig. 1) in media with relative dielectric permittivity larger than $5 .{ }^{27} \mathrm{~A}$ charge transfer complex (CTC) with $\mathrm{C}_{2 v}$ symmetry (as represented in Scheme 1) is predicted in all media. In that work, it was also shown that the solvent effect on the activation barrier is extremely large, in agreement with the experimental variation of the rate constant, and that non-equilibrium solvent effects are quite important.

In the present work, we shall study the process schematized in Scheme 1 in aqueous solution with the help of a hybrid DFT/MM MD technique. Such a study is expected to produce deeper insight into the role of water on charge separation reactions. We shall mainly discuss the dynamics aspects of the reaction and illustrate them with some selected MD trajectories.

The organization of the paper is as follows. In Sec. II we summarize the QM/MM MD method. In Sec. III, we describe the approach to simulate rare event trajectories and the method to select starting configurations for the solvated TS. Computational details are given in Sec. IV and in Sec. V we present and discuss the results. The final section outlines the main conclusions of the work. 


\section{HYBRID QM/MM MOLECULAR DYNAMICS APPROACH}

This approach has been already described in previous publications. ${ }^{10-12}$ Here, we shall outline the main features of the model but the reader can find more details in those papers. In QM/MM models, the total energy of the whole system is divided into three components: the energy of a subsystem described quantum mechanically $E_{\mathrm{QM}}$, the energy of the bulk described at the classical mechanical level $E_{\mathrm{MM}}$ and the interaction between the quantum subsystem and the bulk, $E_{\mathrm{QM} / \mathrm{MM}}$

$$
E_{T}=E_{\mathrm{QM}}+E_{\mathrm{MM}}+E_{\mathrm{QM} / \mathrm{MM}} .
$$

In this expression, $E_{\mathrm{QM}}$ depends on the position of the quantum mechanical nuclei, $E_{\mathrm{MM}}$ depends on the solvent sites and $E_{\mathrm{QM} / \mathrm{MM}}$ depends on both. Both $E_{\mathrm{QM}}$ and $E_{\mathrm{QM} / \mathrm{MM}}$ depend on the electronic density of the quantum subsystem $\rho(r)$ obtained after solving the corresponding Hartree-Fock or Kohn-Sham equations. The Hamiltonian to be used in these equations must include the interactions with the environment, the form of which depends on the type of potential used to describe the classical molecules. Note that $E_{\mathrm{QM} / \mathrm{MM}}$ contains not only the electrostatic part of the interaction but also a crossed van der Waals energy between quantum nuclei and solvent interaction sites. In the calculations presented in this paper we have used the TIP3P potential ${ }^{28}$ to describe the classical water molecules. Therefore, the quantum subsystem Hamiltonian contains the electrostatic potential created by the charges located on the hydrogen and oxygen atoms of the water solvent sites. Polarisable potentials are available and in principle can be implemented in QM/MM simulations. In that case, the Hamiltonian of the solute must also include the interaction with the induced multipole moments. ${ }^{29}$

Forces acting on the quantum or classical system nuclei are obtained analytically from the derivatives of the energy with respect to the corresponding coordinates.

The algorithm of the MD simulation is therefore as follows: (1) get the wave function of the quantum subsystem by solving the Kohn-Sham equations for a given configuration of the whole system; (2) store the new wave function to be used as the SCF initial guess of the next step; (3) compute the forces on quantum and classical nuclei; (4) solve the equations of motion using the proper MD algorithm. The process is repeated using the new positions of quantum nuclei and solvent molecules.

In summary, hybrid DFT/MM molecular dynamics simulations are carried out as classical simulations except that the forces acting on the subsystems (quantum or classical) require the evaluation of the wavefunction describing the quantum part in the presence of the classical part at each simulation step.

\section{CHEMICAL REACTION SIMULATION METHOD}

As mentioned above, the simulation of a reactive trajectory for a process with a large activation barrier (more than a few $k T$ ) cannot be afforded with standard techniques because of the small probability for the chemical system to climb the potential energy barrier. The approach that we follow here is based on the rare event approach ${ }^{13}$ already employed to study reaction dynamics with classical potentials. ${ }^{14}$ The algorithm consists basically on the next steps: ${ }^{1}$

(1) Define an adequate TS structure and for such a structure define the pseudo-normal mode of vibration corresponding to the reaction coordinate.

(2) Perform NVT molecular dynamics simulations for the TS-structure in solution. Only the reaction coordinate must be frozen so that all the other degrees of freedom, i.e. the solvent coordinates but also the reactants translations, rotations and vibrations, are correctly sampled.

(3) From these simulations, select a set of independent configurations for the whole system (reactants and solvent).

(4) For each initial configuration, define a set of random velocities for the chemical system and solvent atoms using a Maxwell-Boltzmann distribution at the requested temperature.

(5) Integrate the equations of motion forward and backward in time until the chemical system reaches the reactants or the products.

(6) Repeat steps (4)-(5) for all the initial configurations so that a statistically representative sample of reactive trajectories is obtained and average properties can be computed.

Some particular aspects connected to our hybrid QM/MM MD calculations are described in more detail in this and the following section. In particular, the choice of the initial conditions, the velocity randomization method and the computation of reactive trajectories is commented in more detail.

\section{A. Choice of initial conditions}

Let us first consider the choice of the initial configurations, i.e., points (1)-(3) above. Definition of the TS structure and reaction coordinate represents one of the major difficulties of the approach since a reaction coordinate is in general defined by a complex combination of internal parameters and has a clear meaning in gas phase only. An approximate reaction coordinate in solution may be defined by assuming the solute-solvent equilibrium hypothesis through the calculation of the potential of mean force (PMF) with MD simulations. However, such calculations are very costly and in general one is constrained to compute the reaction coordinate in the isolated system, evaluate the solvation free energy at each value of the reaction coordinate and obtain the position of the barrier top in solution. Obviously, this approach assumes that there are no important changes in the reaction coordinate in going from gas to solvent. This assumption fails in many cases, such as in the reaction studied below which is not possible in the gas phase, since the interactions with the solvent may modify considerably the potential energy surface. Another option, more general and rather inexpensive, is to use simple solvent-models that allow to evaluate an average value of the solute-solvent interactions through the use of some macroscopic quantities. The free energy surface in solution is directly evaluated and the transition state may be located on that surface without any ap- 
proximation other than that related to the solvent model. The self-consistent reaction field (SCRF) model developed in our group $^{30}$ has been widely employed to study equilibrium solvent effects on chemical reactions ${ }^{31}$ and has been shown to be particularly useful to analyse reaction mechanisms and free energy surfaces. ${ }^{32}$ Since for the process considered here, the reaction coordinate does not have a trivial definition, we have decided to employ the last option. Note that the results obtained with the SCRF model may be refined afterwards through PMF calculations for instance.

The initial configurations are chosen from the results of NVT MD simulations carried out for the TS structure in solution. During these preliminary simulations, it is important to release all the non-reactive degrees of freedom of the chemical system, except of course the reaction coordinate which must be constrained to its TS value in order to prevent the reaction to occur. When the restriction of the reaction coordinate can be mathematically expressed by a set of holonomic constraints, the linearized ${ }^{33}$ or quadratic ${ }^{34}$ form of the method of Lagrange multipliers can be immediately used. In addition, if the constraints are identical with mere fixation of individual interatomic distances, the iterative SHAKE or RATTLE algorithms (see Ref. 34 and references therein) can be applied as well. If the reaction coordinate has been approximated and restricted conveniently, the TS has to exhibit a stable behaviour with respect to all types of fluctuations without breaking or formation of bonds. There are several available NVT algorithms although in general the Nosé thermostat $^{35}$ and the velocity randomization methods ${ }^{36}$ are recommended. ${ }^{1}$ The latter, which has been chosen in this work, samples the various energetical levels by means of NVE dynamics substituting thus the NVT distribution of states. Although there are several procedures of this randomization, ${ }^{34}$ the reselecting of all velocities at once (repeated at equally spaced intervals of time) is considered as the most appropriate one. ${ }^{4(d)}$ Note that because of its random nature and depending on the system, this method can be more sensitive to fluctuations than the Nosé thermostat technique. A comparative study will be reported in due course. ${ }^{37}$ The random selection of velocities may be done assuming a Maxwell-Boltzmann distribution. When rigid molecules are considered (solvent molecules), the randomization is carried out after separating the rotational and translational contributions. The translational velocity of the centre of mass is easily obtained ${ }^{34}$ but the proper treatment of the rotational degrees of freedom is more tricky. Assuming that the distributions of translational and rotational kinetic energies are equivalent, it is possible to select an energy from the corresponding translational energy distribution, choose randomly the space orientation of the angular velocity and renormalize its magnitude so that the rotational energy relative to the centre of mass is equal to the selected one. Concerning the case of flexible molecules (the QM subsystem here) the situation is slightly more complicated since the Maxwell-Boltzmann distribution rigorously holds for the translational motion of their centre of mass only. ${ }^{38}$ In previous studies using classical MD simulations, it was proposed to assign velocities from a Maxwell-Boltzmann distribution for all the degrees of freedom. ${ }^{4(a)}$ This appears to be a good approach for flexible classical molecules but fails when applied to the quantum subsystem because it is hardly adapted to high-frequency vibration modes. One could in principle randomize the rotational and translational degrees of freedom only but unfortunately the vibrational modes and the rotational motion cannot be rigorously separated. The approximation of velocity randomization that we have adopted here for the quantum subsystem takes advantage of the fact that the TS may be schematically represented by a structure $A-B$ where the $A B$ distance is the reaction coordinate (i.e., $A=$ bromonium cation and $B=$ bromide anion). The translational velocities of the centres of mass of $A$ and $B$ are assigned a random value from the Maxwell-Boltzmann distribution preserving thus the velocities of the other degrees of freedom.

The initial configurations are selected from the NVT simulation by storing on disk the coordinates of the whole system at a given frequency, the time between two consecutive configurations being not too small in order to get configurations as independent as possible. This point has been discussed in detail by Gertner et al. ${ }^{4(\mathrm{~d})}$ who proposed to use a procedure in which constant temperature dynamics from 10 independently created seeds are used to generate the initial configurations with velocity randomizations each 250 fs. In $\mathrm{QM} / \mathrm{MM} \mathrm{MD}$ calculations, this procedure would be too costly and the initial configurations can been selected using a single NVT simulation. However, such a simulation must be sufficiently long in order to ensure the proper sampling of the quantum system and solvent. In our work, according to, ${ }^{4(d)}$ the configurations are stored with the same frequency as velocity randomizations (see below). A second randomization is then carried out for these configurations and the corresponding velocities are stored on disk to be used as initial values in the simulation of trajectories.

\section{B. Calculation of trajectories}

After selection of the initial configuration and kinetic quantities, the constraint imposed on the quantum system is removed and a short simulation (typically $500 \mathrm{fs}$ ) is carried out forward and backward in time till the system reaches the reactants or the products. In general, the NVE ensemble is used for these short trajectories although Nosé's NVT dynamics could also be employed. The trajectories can then be classified as reactive, when reactants are connected to products, or non-reactive, otherwise. The latter are a consequence of non-equilibrium solvation and supposes a barrier recrossing (note that reactive trajectories may have also recrossings). In general, backward integration can be simply achieved using a negative time step. Within the family of Verlet algorithms ${ }^{34}$ the change of sign of all velocities, angular momenta and variable $\xi$ of Nosé-Hoover's thermostat $^{35}$ (if used) can work as well. In the case of the "leap-frog" algorithm and derived quaternion formalism, ${ }^{34}$ where kinetic quantities are retarded with respect to 
configurational ones, the corresponding shift in the backward integration must be properly taken into account to avoid discontinuity of resulting joined trajectory in phase space.

Using the final set of reactive trajectories it is possible to make arithmetical averages of all the important quantities at each time step. ${ }^{4(d)}$ It is evident that some fundamental features can be overlooked in this way, for instance when they appear in different trajectories at different times. ${ }^{39}$ Therefore, analysis of individual trajectories is necessary although general conclusions cannot be derived unless the same behaviour appears repeatedly. Interesting analysis may be also carried out for the complementary set of non-reactive trajectories (separated in reactants-reactants and productsproducts trajectories). Finally, the transmission coefficient, which is a fundamental physico-chemical quantity, may be computed and has represented often the final goal of this type of MD simulations.

Before closing this section it is necessary to comment on the choice of the integration step. In principle, its value is dictated by the high-frequency vibration modes related normally to bond stretchings. Therefore, it is desirable to analyze the shortest period of bond oscillations for a proper choice of the time step of integration. Obviously, this time step may be much smaller than the value required for proper integration of low-frequency modes (such as the pseudomode relative to the reaction coordinate) and can be quite computer demanding. Multi-time-step approaches ${ }^{40}$ may be interesting procedures to save computer time but only after adapting them to the QM/MM model. Indeed, computing forces on the quantum system, that may present low and high frequencies of vibration, requires in principle the calculation of the wave function which is the most time-consuming part of the simulation.

\section{COMPUTATION DETAILS}

\section{A. QM and MM potentials}

To describe the classical water molecules we use the TIP3P potential. ${ }^{28}$ This potential assumes rigid water molecules and therefore we do not allow for energy flow into and out of solvent vibrational modes. However, the intramolecular water potential energy of the water molecules was shown ${ }^{4}$ to be constant over the course of a $S_{N} 2$ reaction simulation so that high frequency vibrations of the water molecules did not directly contribute to the activation of the reactants. This hypothesis is assumed here. A more serious limitation of this potential is that the electronic polarization of solvent molecules is not explicitly considered but treated in average. Polarizable potentials may be used in principle but the cost increases considerably and we decided not to employ them in this study.

The quantum system is described using DFT calculations. As stated above, the wave function of the system is computed at each simulation step and therefore it is much computationally time consuming. Obviously, the theoretical level has to be chosen as a hard compromise between computer capabilities and accuracy. The choice requires then a careful preliminary study of the chemical process under con- sideration. Different basis set and exchange-correlation potentials may be employed and the results compared with other $a b$ initio calculations. Since the reaction considered in this work is not possible in gas phase, this comparative study has been carried out in a model solution using the SCRF approach. ${ }^{30}$ A detailed study will be published elsewhere but we give the main conclusions of such a study below (see Sec. IV C). As shown, reasonable results for the reaction are reached using double- $\zeta$ basis sets and polarization functions, with a pseudo-potential for describing non-valence electrons in $\mathrm{Br}$, together with the local spin density (LSD) approximation and the functional due to Vosko, Wilk and Nusair $(\mathrm{VWN}){ }^{41}$ The basis set is that implemented in program deMon, ${ }^{42}$ which has been used for the SCF calculations, with contractions: $\operatorname{Br}(51 / 31 / 41), \mathrm{C}(621 / 41 / 1), \mathrm{H}(41)$. In the deMon code, a second set of auxiliary functions is required $\operatorname{Br}(3,5 ; 3,5), \quad \mathrm{C}(4,3 ; 4,3)$ and $\mathrm{H}(4 ; 4)$. Integration of the exchange-correlation energy was made on a medium grid. ${ }^{42}$ Some test calculations have been also performed with the Becke-Perdew (BP) functional ${ }^{43}$ to test the influence of density gradient corrections on the exchange-correlation terms for the geometries and activation energy.

Note finally that the Lennard-Jones parameters of the quantum system are those proposed in Refs. 44(a) and 44(b) for bromine and ethylene, respectively.

\section{B. Simulations}

After thermalizing the constrained transition state (see below for a description of its structure), a 70 ps simulation was carried out. The NVT ensemble with the velocity randomization method were employed as described above using an integration time step of 1 fs. Randomizations are carried out each $500 \mathrm{fs}$. At the same time the current configuration is stored on disk and a second velocity randomization is performed which completely define the initial conditions of the trajectories to be simulated. A quaternion based leap-frog algorithm due to Fincham ${ }^{45}$ was used for integrating the equations of motion in the classical part and the velocity Verlet algorithm ${ }^{46}$ for the quantum part. In this case, the SHAKE algorithm ${ }^{47}$ was used to keep the reaction coordinate fixed. ${ }^{48}$

Short NVE simulations were carried out starting and the top of the barrier by using the stored set of configurations as initial conditions. The trajectories were integrated forward and backward in time during at least $0.5 \mathrm{ps}$, which is in general enough to reach either the reactants or the products.

All simulations were performed using a cubic box of $20.8 \AA$ of side with 300 classical water molecules, at $25^{\circ} \mathrm{C}$. Periodic boundary conditions and a cutoff distance of $10.4 \AA$ have been applied for all classical-classical and classicalquantum interactions. With these parameters, correlation between periodic images in the first two solvation shells is avoided.

\section{Definition of the reaction coordinate}

As a preliminary study, the first step of ethylene bromination (see Scheme 1) in liquid water has been studied 
TABLE I. Summary of SCRF/DFT calculations for the charge transfer complex (CTC) and transition state (TS) for the reaction of molecular bromine with ethylene (bond lengths in $\AA$, charges in a.u., energies in $\mathrm{kcal} \cdot \mathrm{mol}^{-1}$ ) (Ref. 50).

\begin{tabular}{lccc}
\hline \hline & & VWN & BP \\
\hline$d_{\mathrm{BrBr}}$ & $\mathrm{CTC}$ & 2.51 & 2.58 \\
& $\mathrm{TS}$ & 4.18 & 4.08 \\
$d_{\mathrm{BrC}}$ & $\mathrm{CTC}$ & 2.41 & 2.59 \\
& $\mathrm{TS}$ & 2.08 & 2.08 \\
$q_{\mathrm{Br}^{-}}$ & $\mathrm{CTC}$ & -0.336 & -0.326 \\
$\Delta E^{\text {act }}$ & $\mathrm{TS}$ & -0.927 & -0.926 \\
\hline \hline
\end{tabular}

through DFT calculations using the SCRF model for the solvent $(\epsilon=80.0)$, the VWN (41) and BP (43) functionals and a double- $\zeta$ plus polarization basis set, as described above. Some results are summarized in Table I. In both cases, the results predict the formation of CTC and TS structures in water having both $C_{2 v}$ symmetry.

Though a previous study for related CTC's in gas phase $^{49}$ has shown the difficulties of DFT to reproduce experimental geometry, especially at the local level, no DFT result is available for the corresponding TS structures. Since the TS is much more polar than the CTC, the electrostatic interactions dominate and the influence of density gradient corrections on the geometry is expected to be smaller in that case. Actually, for the TS, the results are similar in VWN and $\mathrm{BP}$ calculations. The $\mathrm{BrBr}$ distance is large and the charge transfer is almost complete. The main difference in the computed geometries appears for the $\mathrm{BrBr}$ distance which is slightly larger at the local level but the agreement can be considered reasonably good. The transition vector, i.e. the eigenvector corresponding to the only imaginary frequency at the TS is essentially defined (98\%) by the $\mathrm{BrBr}$ interatomic distance. ${ }^{50}$

The difference in the activation energies calculated using the BP and VWN functionals are slight presumably by cancellation of errors. A warning is necessary here. The use of gradient-corrected functionals could be crucial if solvent water molecules were described quantum mechanically. This is not the case here but arises in ab initio molecular dynamics using the Car-Parrinello algorithm. ${ }^{52}$ Thus gradientcorrected functionals has been shown to be important in simulations of liquid water. ${ }^{53}$ In our study, in which solvent molecules are described classically, accounting for gradient corrections would improve a little the description of the solute but would imply a substantially larger cost. Therefore, we decided to employ the VWN functional which appears to correctly describe the main features of the model charge separation reaction considered in the present application.

\section{RESULTS}

\section{A. Transition state equilibrium solvation}

Before discussion of reactive trajectories, it is interesting to analyze in some detail the NVT simulation for the TS structure in water solution that has been used to define the

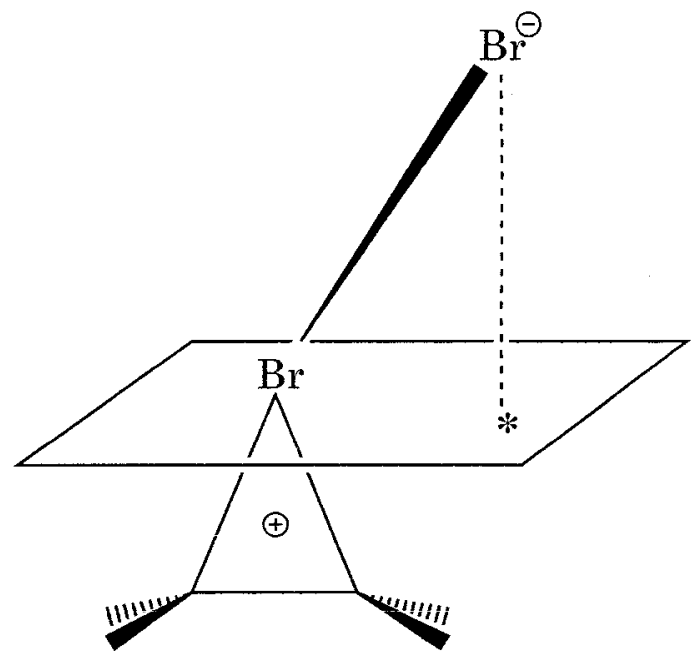

$\mathrm{X}(\mathrm{A})$

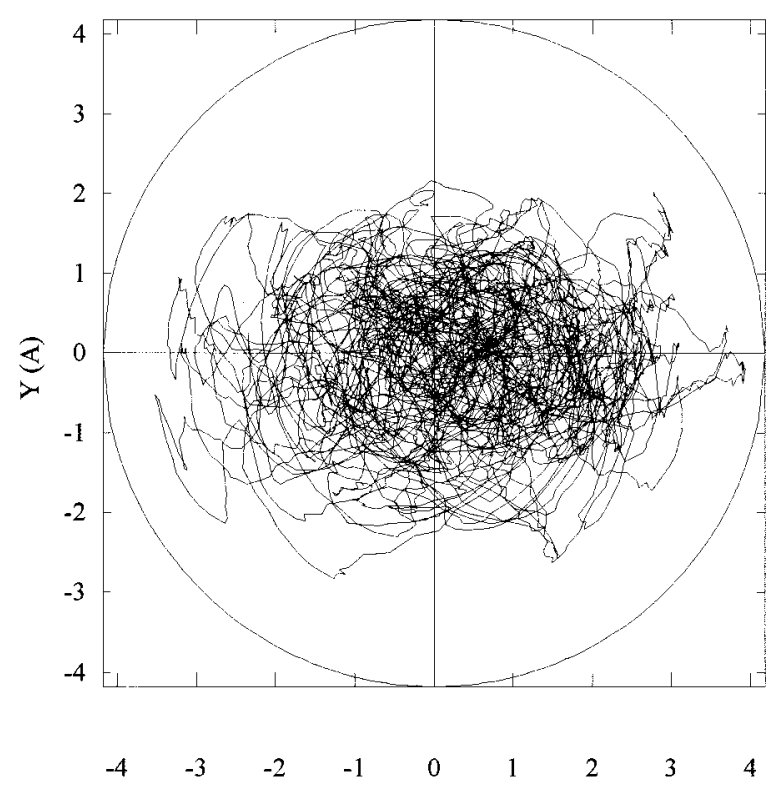

FIG. 2. Projection of the $\mathrm{BrBr}^{-}$vector on a plane perpendicular to the axis defined by the $\mathrm{Br}$ atom in bromonium and the centre of the $\mathrm{CC}$ bond (top) along the $70 \mathrm{ps}$ simulation for the constrained transition state (bottom).

initial conditions. Note again that the TS structure has only one constraint, the $\mathrm{BrBr}$ interatomic distance. All the other parameters are free to change during the simulation including the $\mathrm{BrBrC}$ angles since they do not participate primarily in the reaction coordinate definition. In other words, the structure is not constrained to have any particular symmetry. We predict indeed large fluctuations of the system with respect to the average $C_{2 V}$ symmetry. This is illustrated in Fig. 2 where the $\mathrm{BrBr}^{-}$vector is projected on a plane perpendicular to the axis defined by the $\mathrm{Br}$ atom in bromonium and the centre of the CC bond (see Fig. 2). Obviously, the mean value preserves the $\mathrm{C}_{2 \mathrm{~V}}$ symmetry. Though the forming anion may lie far from the symmetry axis, there is a large probability to find it on the axial region. The large 

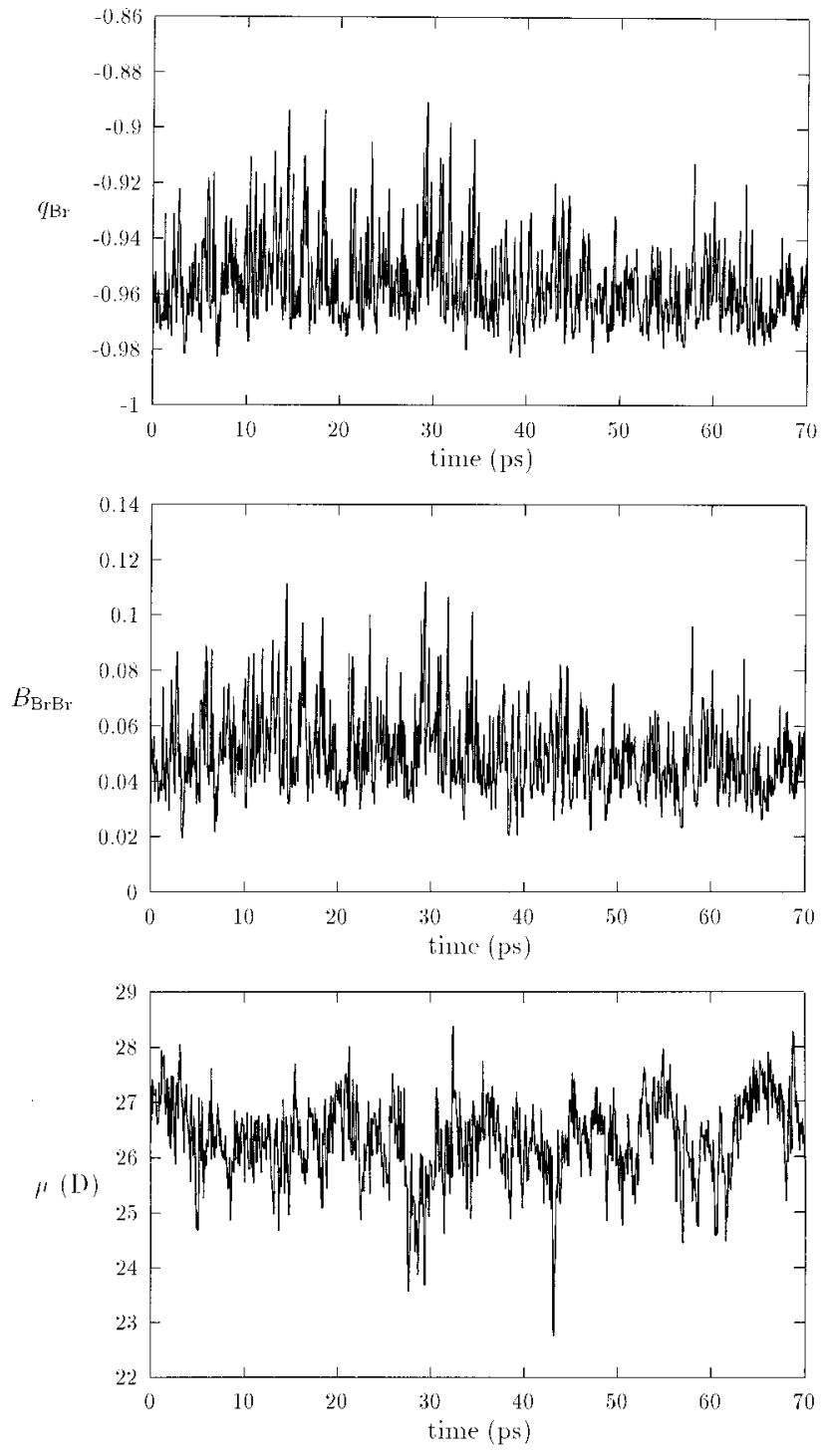

FIG. 3. Fluctuations of the forming bromide anion charge $\left(q_{\mathrm{Br}}\right)$, BrBr Mayer bond order $B_{\mathrm{BrBr}}$ and dipole moment $\mu$ of the quantum system along the 70 ps NVT simulation of the constrained transition state.

amplitude of these deformations reveals the presence of a flat potential energy surface. The main consequence is that many reactive events will cross the barrier through a TS structure which is substantially distant from the hypothetical $\mathrm{C}_{2 \mathrm{~V}}$ symmetry.

Another striking result concerning this simulation is the fluctuation of the electronic charge distribution on the chemical system. The net Mulliken atomic charge on the forming $\mathrm{Br}^{-}$, the $\mathrm{BrBr}$ bond order and the TS dipole moment are represented in Fig. 3 as a function of time. The first quantity varies between 0.980 and 0.890 . The $\mathrm{BrBr}$ bond order oscillates between 0.020 and 0.110 and the dipole moment, which has a large magnitude due to the fact that the TS is essentially described by an ion pair, lies roughly between 25 and $28 \mathrm{D}$ although smaller values may be randomly observed.

The radial distribution functions (RDF's) for the outmost atoms in the TS are given in Fig. 4. In the case of the form-
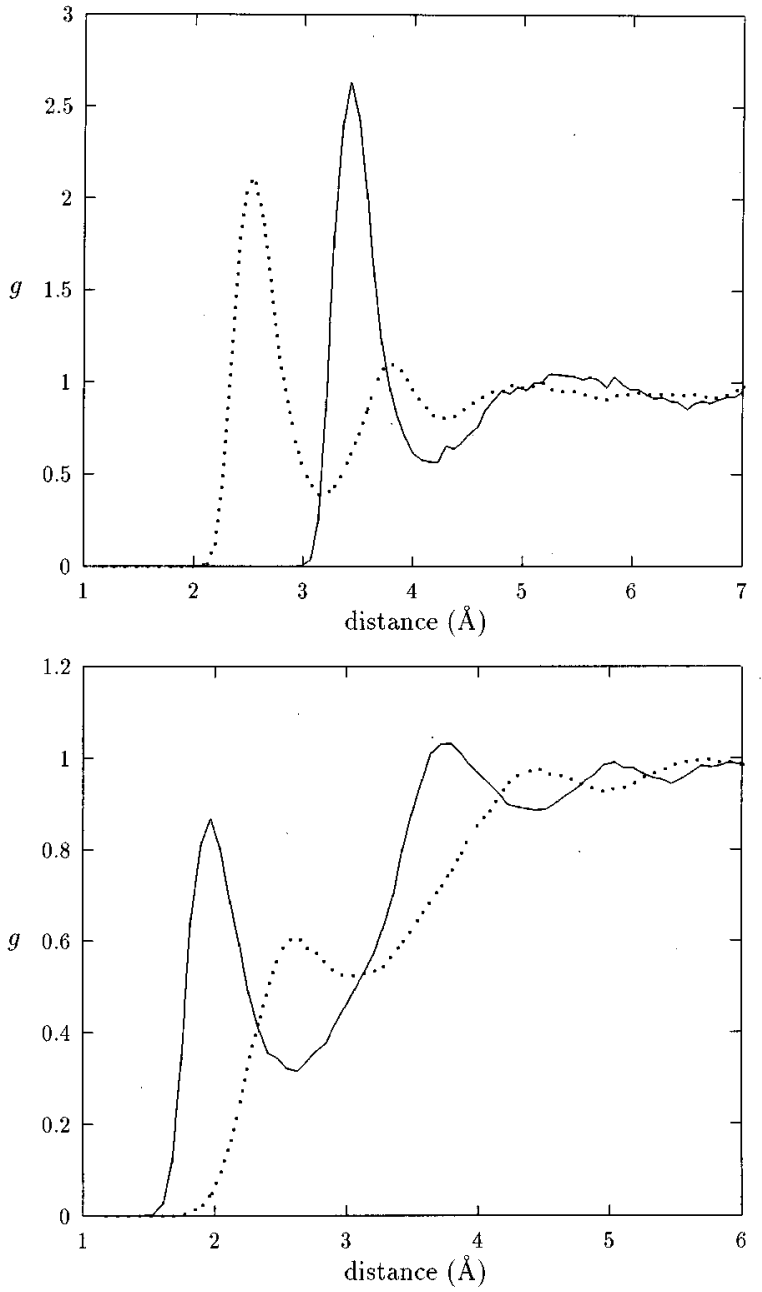

FIG. 4. Radial distribution functions $g_{X Y}$ obtained in the $70 \mathrm{ps}$ simulation for the constrained transition state ( $X=$ quantum system atom, $Y=$ solvent atom). Top: $\mathrm{BrO}$ (full line) and $\mathrm{BrH}$ (dotted line). Bottom: HO (full line) and $\mathrm{HH}$ (dotted line).

ing bromide anion, the $\mathrm{BrO}$ and $\mathrm{BrH}$ RDF's are very close to those obtained for the bromide anion in water using a similar DFT/MM simulation. ${ }^{54}$ The position of the first peaks is 3.43 and $2.48 \AA$, respectively, whereas the coordination number of the forming bromide anion with oxygen atoms is 7.50. The same quantities reported for bromide in water using hybrid DFT/MM MD simulations are: ${ }^{54}$ peaks at 3.45 and 2.52 $\AA$ and coordination number equal 7.7 (the experimental coordination number for the same system is 6.3) ${ }^{55}$ Figure 4 displays also results for the RDF around the hydrogen atoms in ethylene. In the TS, these hydrogen atoms carry a substantial part of the total positive charge on the forming bromonium cation. Therefore, noticeable interactions with solvent water molecules are expected and this is confirmed by the form of the corresponding RDF's. As seen in Fig. 4, there is a net $\mathrm{HO}$ band with maximum at $1.97 \AA$.

\section{B. General description of trajectories}

In conventional transition state theory, there are no recrossings of the activation barrier: a trajectory reaching the 
TABLE II. Summary of reactive and non-reactive trajectories. Total number of trajectories presenting $n$ crossings of the barrier according to their type ( $r$ and $p$ hold for reactants and products, respectively). The total number of trajectories is 140 .

\begin{tabular}{crrcc}
\hline \hline$n$ & $r-r$ & $r-p$ & $p-r$ & $p-p$ \\
\hline 1 & 0 & 39 & 0 & 0 \\
2 & 77 & 0 & 0 & 4 \\
3 & 0 & 2 & 5 & 0 \\
4 & 12 & 0 & 0 & 0 \\
5 & 0 & 0 & 0 & 0 \\
6 & 0 & 0 & 0 & 0 \\
7 & 0 & 0 & 1 & 0 \\
\hline \hline
\end{tabular}

TS from the reactants is assumed to proceed through the products. Moreover, the reactants are in equilibrium with the solvent all along the reaction path. Our results show that these assumptions cannot be applied to the process under study. In particular many barrier recrossings are displayed by the trajectories.

A summary of trajectory characteristics including the number of barrier crossings is presented in Table II. The molecular dynamics simulations show many non-reactive trajectories (66\% of the total number) most of them being of the reactants-reactants type (96\%) and only a few (4\%) connecting products to products. The rest (34\% of the total number) are reactive trajectories that in a few cases (15\%) present barrier recrossings. Note that only one reactive trajectory present more than three barrier crossings.

\section{Reactive trajectories}

The trivial classification of reactive trajectories is based on the number of recrossings since this plays a major role in the transmission coefficient. However, in the present case, it is also interesting to consider another classification based on the shape of the function $r(t)$, where $r$ is the chemical system reaction coordinate, the $\mathrm{BrBr}$ distance here. Such an attempt will be valuable in understanding the reaction mechanism in solution. Accordingly, two main types may be distinguished.

Type I is the most frequent and corresponds to trajectories for which the $\mathrm{BrBr}$ distance oscillates first and then continuously increases in time along the path connecting reactants to products. Therefore, no recrossings are observed for this type of trajectories. It is illustrated in Fig. 5 by one particular trajectory that has been followed during 6 ps. In this figure, we have also plotted the variation with time of other representative internal coordinates, namely the $\mathrm{CC}$ and $\mathrm{BrC}$ bond lengths. One may note the oscillations of the $\mathrm{BrBr}$ bond in the reactant side that correspond to vibrations in the stable CTC. Though we have not investigated the stability of this complex, which in principle can separate into an ethylene and a bromine molecules with a low activation barrier, Fig. 5 shows a stable CTC complex during a few picoseconds. The CC curve shows a substantial lengthening for this bond during the activation process that reaches virtually its final length (corresponding to a single $\mathrm{CC}$ bond in a threemembered ring) slightly before the TS. Concerning the $\mathrm{BrC}$
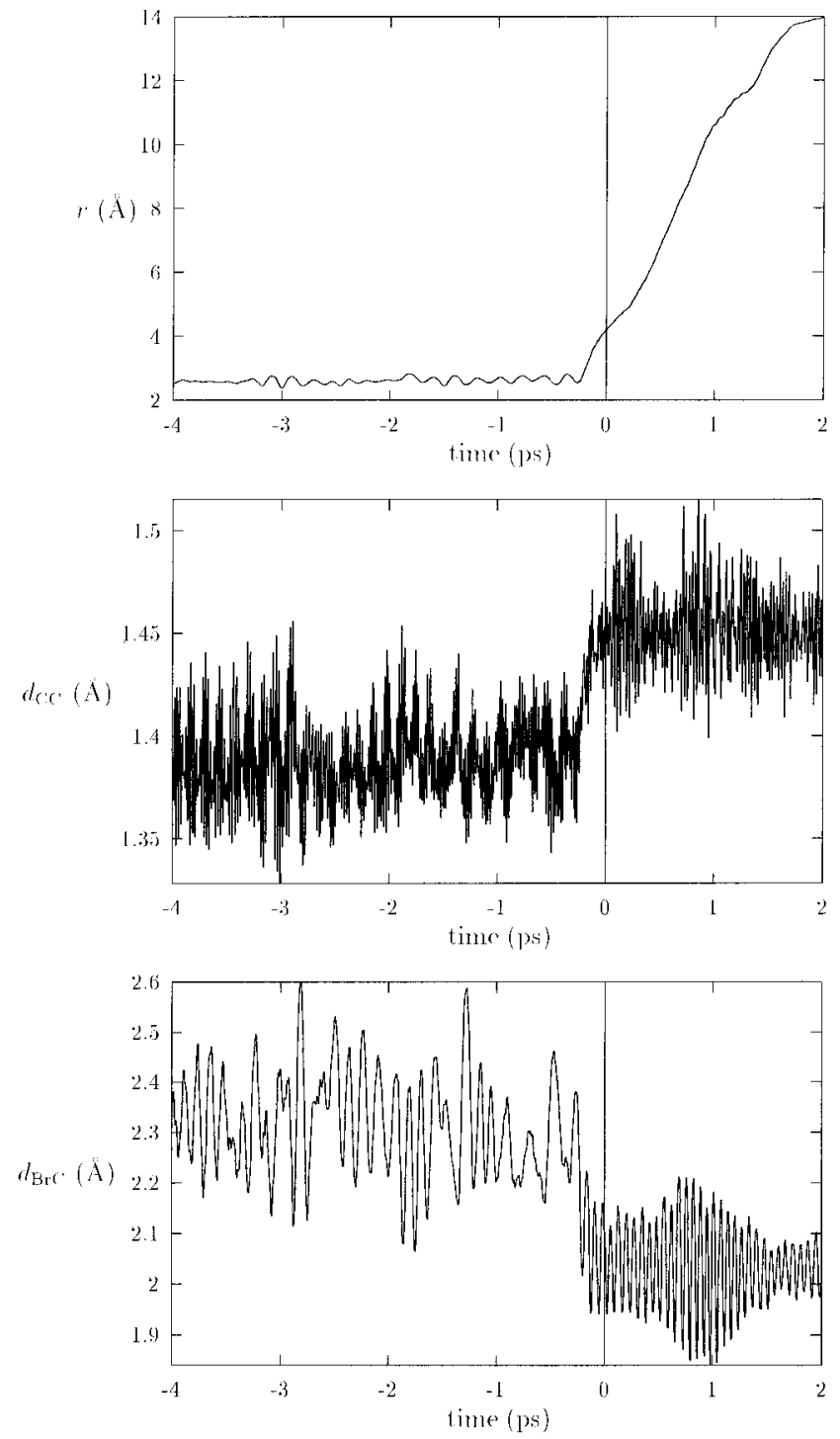

FIG. 5. Evolution of the $r$ coordinate ( $\mathrm{BrBr}$ distance), $\mathrm{CC}$ and $\mathrm{BrC}$ bondlengths in a standard Type I reactive trajectory. The simulation is started at $t=0$ forward and backward in time.

curves, note that the interatomic distances decrease in the activation process due to the formation of the single $\mathrm{BrC}$ bonds. As expected, the frequency of vibration of this bond undergoes a large change in going from the reactants to the product side.

Another representation of the trajectory in Fig. 5 is given in Fig. 6 that illustrates some other interesting features. The position of the forming bromide anion remains close to the "symmetry" axis in the prereactive charge transfer complex. Of course, it oscillates around the hypothetical $C_{2 v}$ structure but the amplitude of these oscillations is not very large in contrast with the corresponding amplitude in the TS (see Fig. 2). Note also in Fig. 6 that in going from the CTC to the TS and then to the products, the position of the bromide anion departs considerably from the axis. This result has been found quite frequently, the hypothetical dissociation along the axis being little probable. 

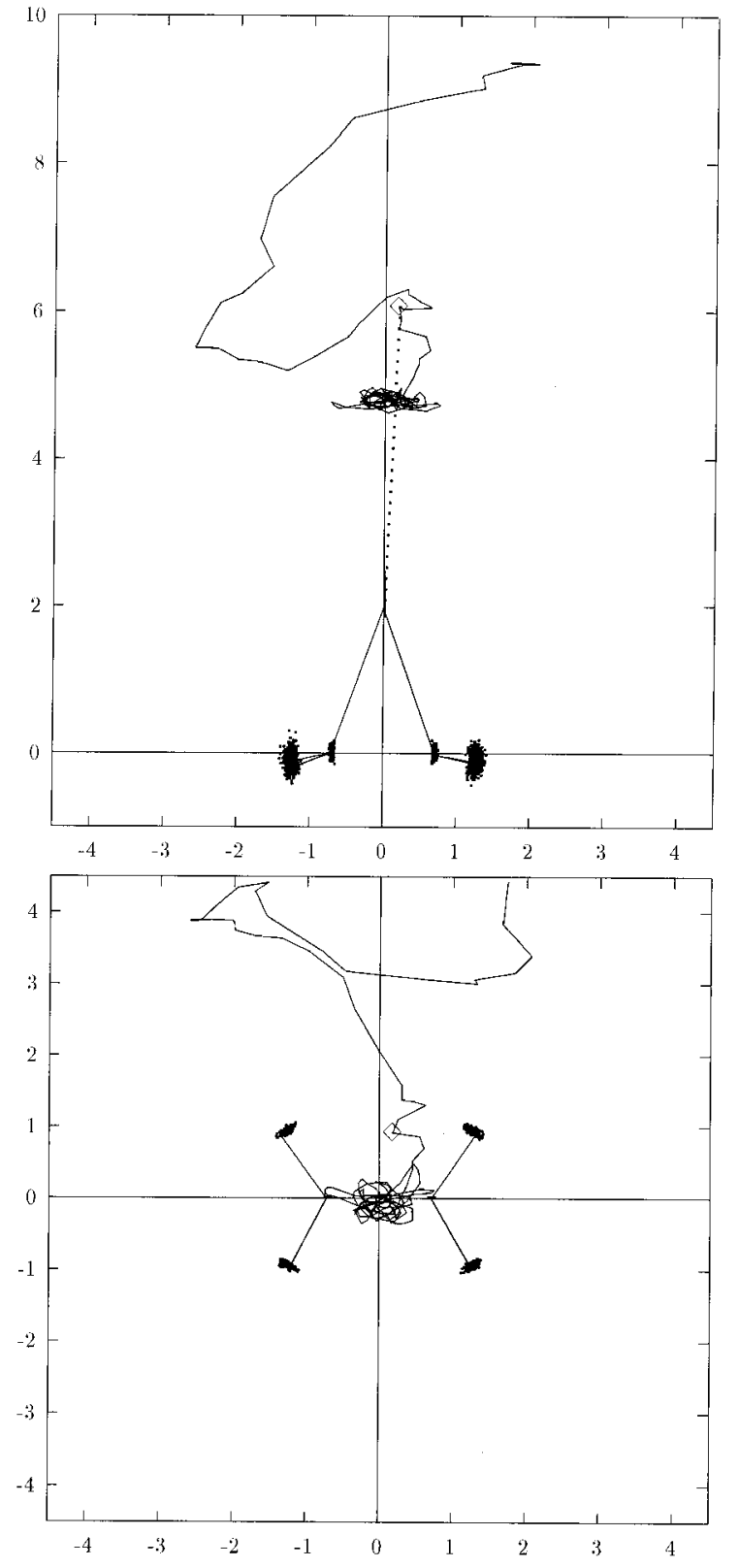

FIG. 6. Projection of the system coordinates along the trajectory represented in Fig. 5 into the $Z Y$ and $X Y$ planes.

The standard $r(t)$ curve for the ethylene bromination reaction may be compared with the corresponding curve for the $S_{N} 1$ reaction of $t-\mathrm{BuCl}$ in classical MD simulations. ${ }^{3}$ First, we do not observe any increase of the oscillations amplitude before the TS, as noted in those calculations. This may be due to differences on the chemical nature of the systems but also to the neglect of any internal degrees of freedom in the $t$-Bu fragment of the $S_{N} 1$ simulation. Second, in our case $r(t)$ increases with time whereas in the $S_{N} 1$ reaction a rapid relaxation of the reaction system toward equilibrium in an ionic well was pointed out. We show below that a related situation holds for certain trajectories of Type II.

Some electronic properties of the quantum system along
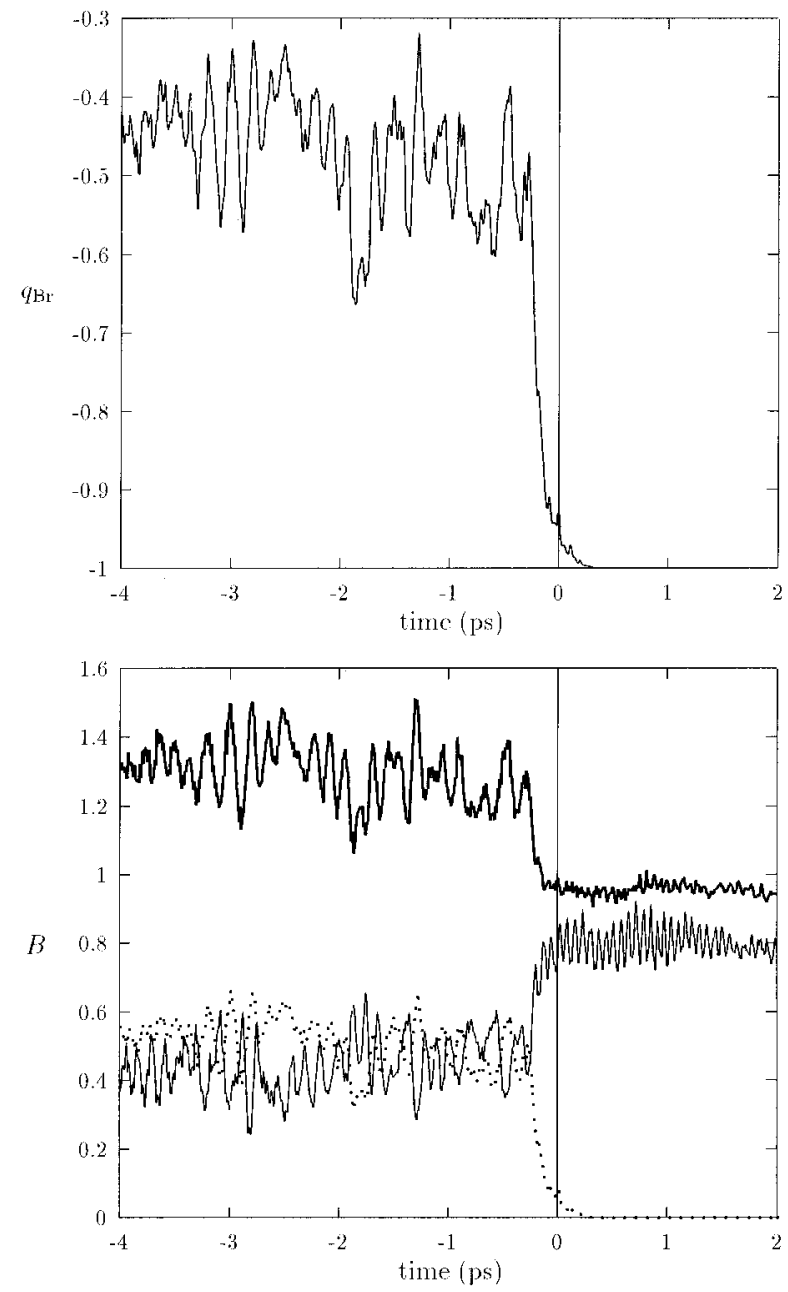

FIG. 7. Evolution of the forming bromide charge and Mayer bond orders $B$ [CC (bold line), $\mathrm{BrC}$ (single line) and $\mathrm{BrBr}$ (dotted line)] along the trajectory represented in Fig. 5.

the trajectory in Fig. 5 are shown in Fig. 7. One may note that the Mulliken charge on the forming bromide anion oscillates between -0.6 and -0.3 in the CTC and decreases rapidly near the TS, where the formation of the bromide anion is almost achieved. The $\mathrm{BrBr}$ and $\mathrm{CC}$ bond orders follow closely the oscillations of this Mulliken charge. Note however that the CC bond order present large oscillations before the TS but only very small oscillations afterward. This contrasts with the CC length oscillation amplitude that does not change a lot after reaction. Note finally that the $\mathrm{BrC}$ bond orders reach also their final value near the TS, the frequency of the oscillations being larger and the corresponding amplitude smaller after formation of the single bonds. All these data show that basically, the bromonium cation and the bromide anion are already formed at the TS, or in other words the TS has a marked late character. This is not surprising considering the large value of the $\mathrm{BrBr}$ bondlength at the TS. Experimentally, there is evidence that considerable charge separation occurs in the activation step ${ }^{18}$ in agreement with the theoretical result.

It is now interesting to estimate the time required to 


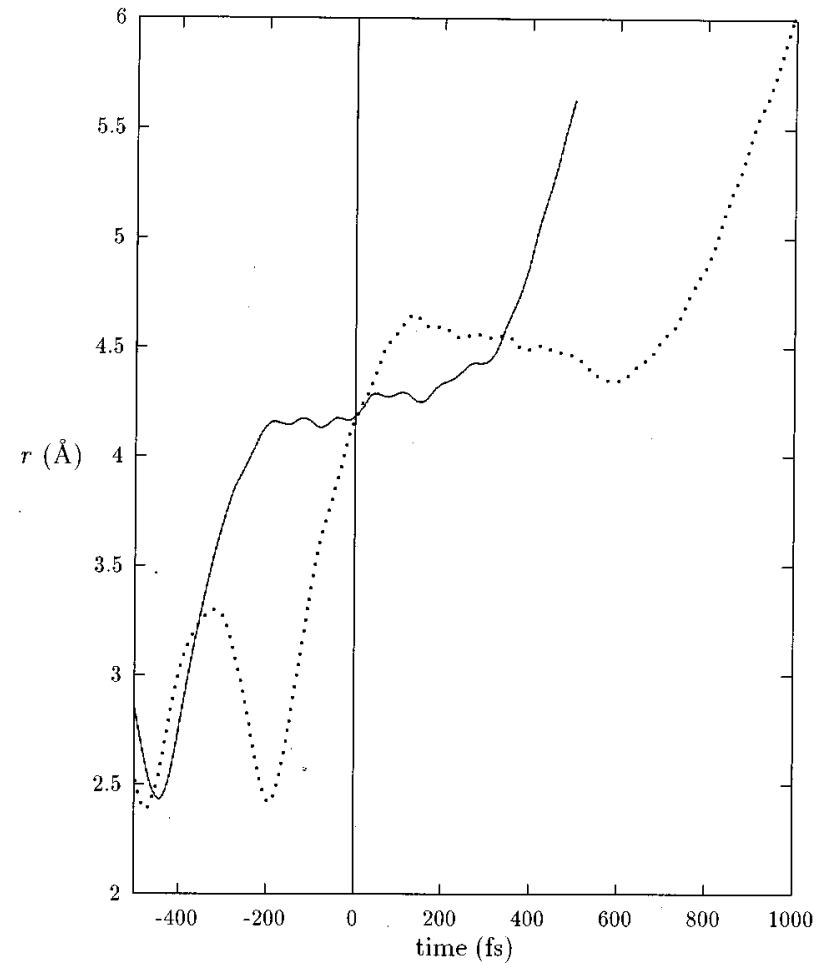

FIG. 8. Evolution of the $r$ coordinate ( $\mathrm{BrBr}$ distance) in representative reactive trajectories of Type IIa (full line) and Type IIb (dotted line). The simulation is started at $t=0$ forward and backward in time.

reach the reactants in Type I trajectories initiated at the TS. For that, we shall consider that the reactants are reached at the first $\mathrm{BrBr}$ oscillation minimum in the reactant side. This times varies in the range 200-300 fs for the ensemble of Type I trajectories. We shall refer to it as the activation time.

Type II trajectories are illustrated in Figure 8. The characteristic feature of these trajectories is that the $\mathrm{BrBr}$ bond length remains almost constant during a non-negligible period of time in the TS region (Type IIa) or slightly after (Type IIb). In fact, the chemical system may stay in an essentially frozen configuration during times as large as 200$500 \mathrm{fs}$. The time of activation (estimated as before) in IIa trajectories varies now in the range 300-500 fs whereas in IIb trajectories it is close to that found for Type I trajectories.

Type IIa trajectories correspond to a TS trapped in a well. The bromonium-bromide ions are trapped in a solvent cage and wait until appropriate fluctuations of solvent molecules allow them to form the reactants (CTC) or the products (separated ions). During that time, the $\mathrm{BrBr}$ distance does not change much but the calculations reflect important angular oscillations. This situation is comparable to that found in model $S_{N} 2$ reactions which was described as a polarization caging regime $(4 c)$ and is a typical nonequilibrium solvation effect. It arises when the nonadiabatic energy profile presents a minimum rather than a maximum at the TS giving rise to a pseudo-stable structure. In IIb trajectories, the $\mathrm{BrBr}$ bondlength for the corresponding pseudostable structure is a little larger than the TS value so that strictly speaking one cannot call it a trapped TS structure. It

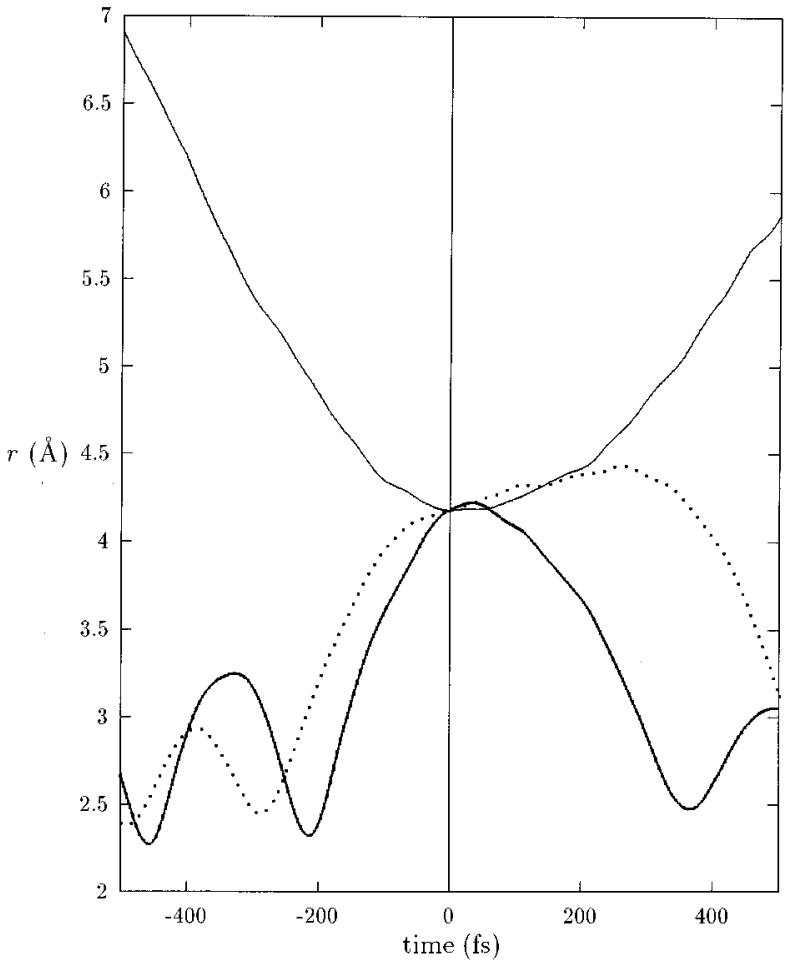

FIG. 9. Evolution of the $r$ coordinate $(\mathrm{BrBr}$ distance) in some non-reactive trajectories. Bold line: reactant-reactant trajectory. Single line: productproduct trajectory. Dotted line: reactant-reactant trajectory with a trapped structure

could be related to contact ion pairs which have been pointed out before in studies for the intimately related $S_{N} 1$ reaction in polar media. Indeed, a shallow ionic minimum on the free-energy surface appears slightly after the TS in those cases. ${ }^{3,56}$ Since we have not computed the free energy profile for the reaction, we cannot confirm the existence of such a minimum in the present case but the analysis of the trajectories tends to show that some structures beyond the TS might have significant lifetimes.

\section{Non-reactive trajectories}

Non-reactive trajectories are illustrated in Fig. 9. Most of them are characterized by a fast recrossing of the barrier after reaching the TS which occurs in a few femtoseconds. As noted above, very few products-products trajectories are found. Trapped structures around the TS are also present in a number of non-reactive trajectories. Such trajectories may be associated to cage reactions (although in the present case, of course, the reactants and products would be the same chemical species). Cage reactions arise because there is an activation energy for diffusion so that the pair of ions (or radicals) collide with each other several times before they separate and recombination may occur during these collisions.

From these results it appears clearly that the destiny of a given trajectory cannot be determined before a substantial period of time after the transition state. In some cases, the trajectory fate remains uncertain during 500 fs after the TS. This contrasts with the results reported for the $S_{N} 2$ reaction 


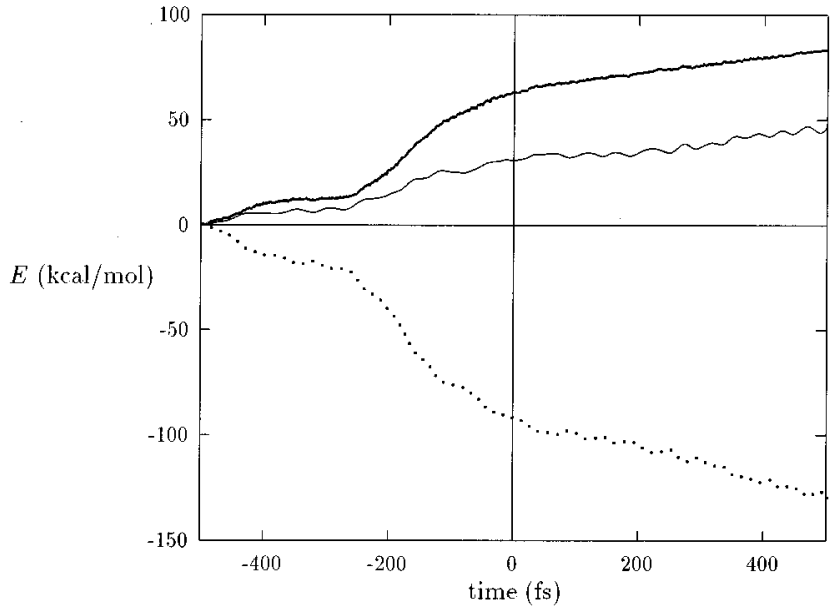

FIG. 10. Average value of the total energy of reactants (bold line) and solvent molecules (single line) and interaction energy (dotted line) over the reactive trajectories.

for which the trajectory fate is determined within \pm 50 fs of the reagents being at the transition state, although in that \pm 50 fs the reagents can cross the transition state more than once. $^{4}$

\section{Energy flow and activation to the transition state}

The average values for the total energy of the quantum reactant system (potential+kinetic), total energy of classical water molecules and the water-reagents interaction energy as a function of time are given in Fig. 10. The evolution of potential and kinetic energies are plotted on Figs. 11 and 12, respectively. In these figures, the average quantities have been obtained using all the reactive trajectories and are given with respect to their value at $t=-500 \mathrm{fs}$ where the trajectories are assumed to be in the reactant side.

Looking at Fig. 10, one sees that the total energies for the reactants and the solvent increases from $t=-500 \mathrm{fs}$ to $t=+500 \mathrm{fs}$ whereas the interaction energy decreases. A pla-

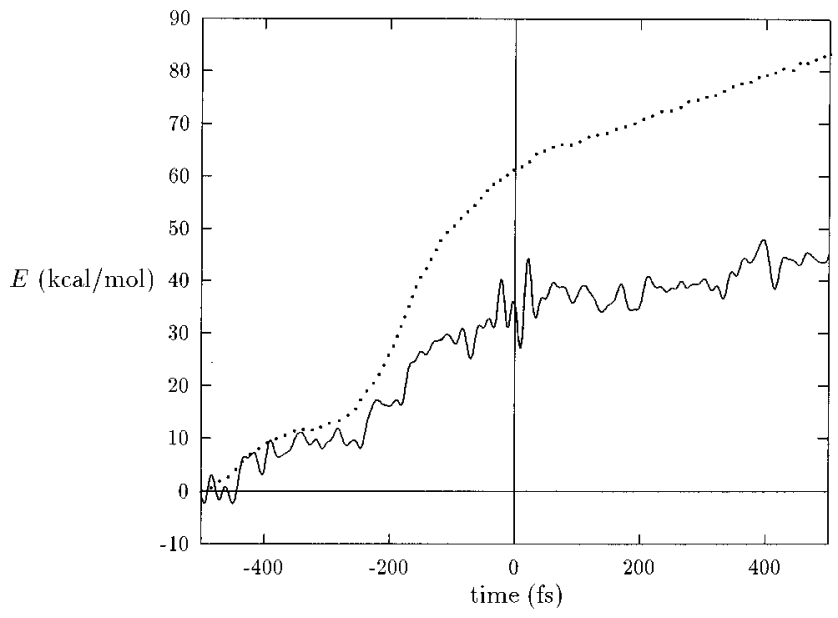

FIG. 11. Average value of the potential energy of the reactants (dotted line) and solvent molecules (single line) over the reactive trajectories.

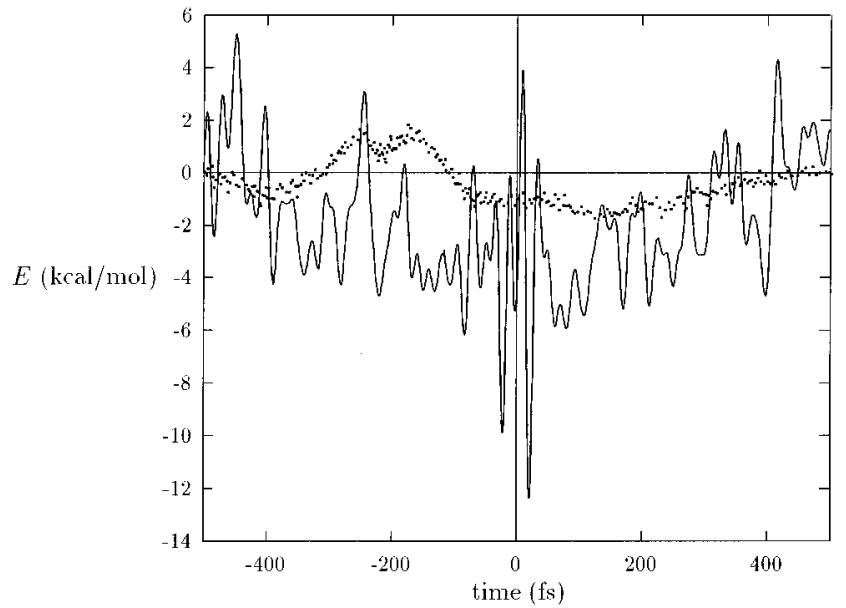

FIG. 12. Average value of the kinetic energy of the reactants (dotted line) and solvent molecules (single line) over the reactive trajectories.

teau is observed in all cases in the -400 to -250 fs region the origin of which may be found on the characteristics of Type I and Type II trajectories. We have noted above that the time of activation (see before) is essentially the same for Type I and Type IIb trajectories and may be roughly estimated to $200 \mathrm{fs}$. On the other hand, for Type IIa trajectories, i.e., those presenting a TS trapped in a well, the activation time is larger and is roughly $400 \mathrm{fs}$. Thus the energy begins to increase at $-400 \mathrm{fs}$ for some trajectories whereas almost all the trajectories have an increasing energy between -200 $\mathrm{fs}$ and $t=0$. Note that the difference $(200 \mathrm{fs})$ correspond basically to the inverse of the $\mathrm{BrBr}$ oscillation frequency in the CTC. This is clearly shown in Fig. 8. The first BrBr bond length minimum (in going from TS to reactants) for the Type IIa trajectory matches the second minimum for the Type IIb trajectory (Type I trajectories are analogous to Type IIb in the reactant side).

Quite noteworthy is the fact that the total relative solvent energy is approximately equal to half the total relative solute energy along the simulation. Obviously, since reactive trajectories are obtained in the NVE ensemble (although the initial configurations were obtained in the NVT ensemble for the solvated TS), the increase in total reactant and solvent energies is compensated by the interaction energy term so that total energy is conserved. Thus the interaction energy variation is roughly equal to $3 / 2$ the variation on total solute energy.

One may wonder whether the solvent is almost frozen during the crossing of the transition barrier. In that case, the energy might remain unchanged during a few femtoseconds around the TS. In Fig. 10, there is not evidence of such an effect (note that curves in this Figure represent average quantities) in contrast to the results reported for other reactions. ${ }^{4(\mathrm{~d})}$ This may be explained if one considers the contribution from Type IIa trajectories, for which the pseudostable TS allows solvent reorganization during the barrier crossing.

The variation of reactants potential energy in Fig. 11 resembles much the variation of the total energy because the 


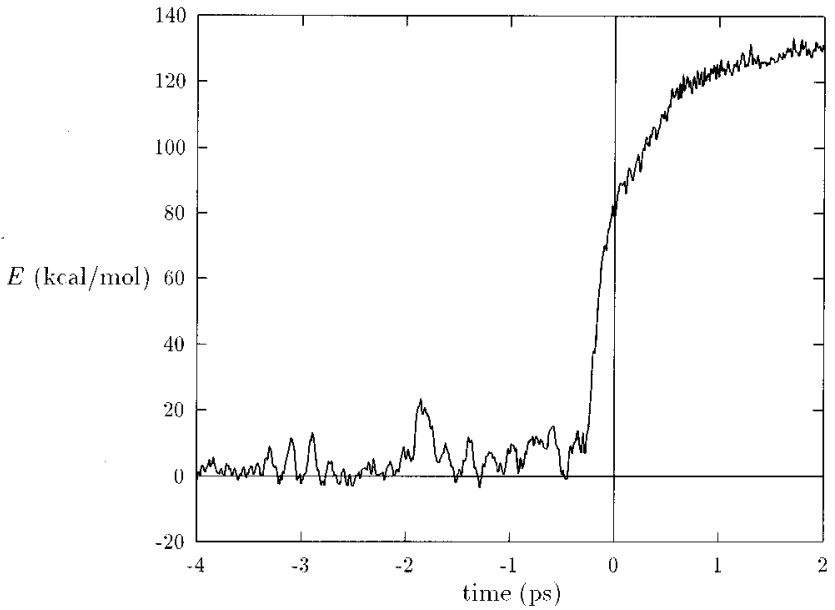

FIG. 13. Evolution of the total energy of the reactants along the standard reactive trajectory in Fig. 5.

kinetic energy changes for the reactants are relatively small. Thus the total change in potential energy in the period of time considered is ca. $85 \mathrm{kcal} / \mathrm{mol}$ for the reactants whereas the maximum difference in kinetic energy in the same period are below $3 \mathrm{kcal} / \mathrm{mol}$, as visible in Fig. 12. The large variation of the reactants potential energy is not surprising since the reaction is not possible in gas phase. The largest increase appears between $t=-200 \mathrm{fs}$ and $t=0$ but the potential energy continues to increase even at times as large as $t=+500$ because of the $1 / r$ behavior of the electrostatic potential between the bromonium and the bromide ions. In Fig. 13 we show the potential energy variation for a particular trajectory (that in Fig. 5) up to 2 ps above the TS, illustrating the slow convergence of the term. The main feature exhibited by the reactant kinetic term variation (see Fig. 12) is a net increase at ca. $t=-200 \mathrm{fs}$ followed by a decrease in reaching the TS. Then, it oscillates from $t=-100$ fs to $t=+200$ fs approximately and begins to increase again. This behavior may be a consequence of the polarization caging regime in Type II trajectories in which the reaction coordinate changes slowly during 200-500 fs. Since this term is small, our description cannot be quantitative here and a larger number of reactive trajectories should be necessary. In addition, a larger simulation time before and after the TS would be needed.

In the case of the solvent, the potential and kinetic energy terms exhibit appreciable fluctuations. The potential energy of the solvent increases (again a factor $1 / 2$ is found with respect to the solute potential energy variation). This increase is due to the fact that the solvent must be ordered around the forming ions in order to solvate them. Apart from an entropic unfavorable contribution (not considered here), the repulsive part of the interaction between the water molecules is enhanced. This is an orientational-polarization energy which is naturally compensated by the stabilizing solute-solvent interaction energy but also by a decrease of the solvent kinetic energy around the TS as shown in Fig. 12.

As the chemical system proceeds from reactants to products, the water molecules must reorient around the forming ions. In the reactants, the charge separation is still moderate

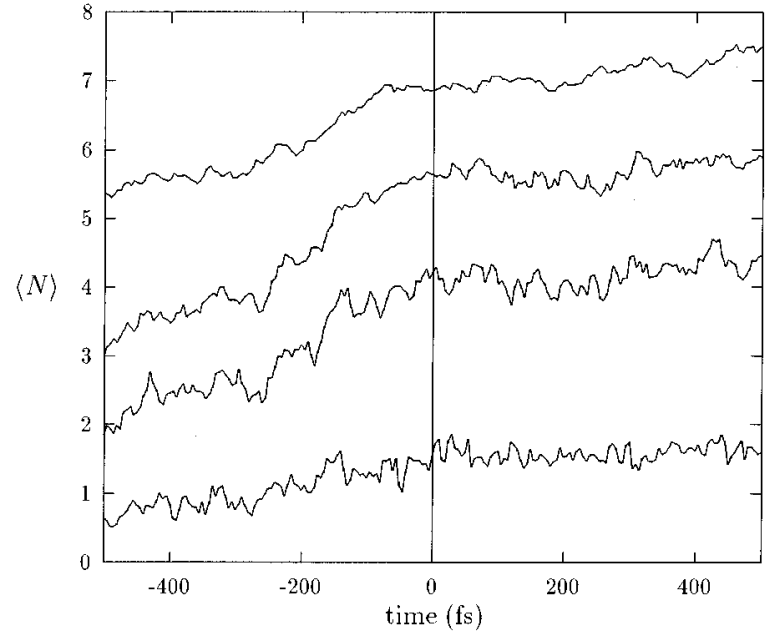

FIG. 14. Average number of water molecules "bonded" to the external bromine atom along the reactive trajectories as a function of the maximum value of the $\mathrm{BrOH}$ angle considered (see the text for more details). From top to bottom, $\mathrm{BrOH}=180^{\circ}, 30^{\circ}, 20^{\circ}, 10^{\circ}$.

and any large solute-solvent interaction is expected. In the products, two ions are formed and therefore large solutesolvent interactions are present. The transition state is an intermediate structure but we have seen that it is already quite polar and the RDF around the forming bromide is very close to that found previously for a bromide anion in water. The evolution of the first water shell along the reaction is therefore interesting. In Fig. 14, we plot the average number of water molecules "bonded" to the external bromine atom. This number $N$, is determined by geometrical factors simply. We carry out a first test on $\mathrm{BrO}$ distance, which must lie between 3 and $4 \AA$ (i.e., the limits of the first peak in the bromide $\mathrm{RDF}$ for $\mathrm{BrO}$ ), and a second test on the $\mathrm{BrOH}$ angle, which must be smaller than a given value. Plots of $\langle N\rangle$ for different values of this angle are done in Fig. 14

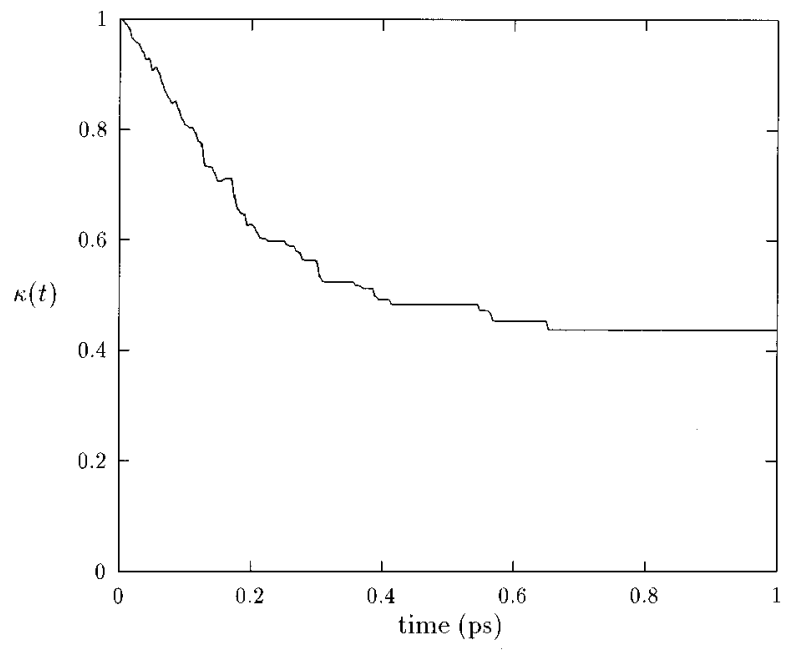

FIG. 15. Time-dependence of the transmission coefficient $\kappa(t)$. 
(the average is done over reactive trajectories). Obviously, the curves depend on the angle employed since $N$ increases for larger angle values. In the limit $\left(\mathrm{BrOH}=180^{\circ}\right),\langle N\rangle$ would fit the number obtained from integration of the $\mathrm{BrO}$ RDF band. Independently of the angle chosen, $\langle N\rangle$ grows from $t=-500$ fs to $t=0$ where the value is stabilized. In other words, the number of first-shell water molecules solvating the bromide anion in the TS is practically the same that the number of solvating molecules for a simple bromide anion in water. This is consistent with the close similarity between the first peak of the corresponding RDF noted above. The increase in the reactant side is not made steadily. There is a moderate increase between -500 and -250 fs followed by a rapid increase till reaching the TS at $t=0$. This variation in the negative times corresponds roughly to that exhibited by the interaction energy. After crossing the barrier, however, the interaction energy continues to increase (in absolute value) whereas the first solvation shell of the bromide remains approximately constant. Therefore, after the TS, the main modifications in solvent structure concern further shells. Probably, the same trend holds for the bromonium cation but the analysis is more complex and we did not try to do it.

\section{Transmission coefficient}

The computation of the transmission coefficient $\kappa$ is quite interesting since it gives a direct information on the role of dynamic solvent effects. It represents the correction to the TST rate constant $k^{\mathrm{TST}}$ due to recrossings of the energy barrier

$$
\kappa=k / k^{\mathrm{TST}},
$$

where $k$ is the true rate constant. Different expressions may be used to compute this coefficient. Here, we have used a "positive flux" formulation ${ }^{57}$ that assumes that the trajectories are initiated at the barrier top with forward momentum along the reaction coordinate so that the system is always in the product side at sufficiently small positive times and in the reactant side at sufficiently small negative times. Therefore, for a given time $t$, the time-dependent transmission coefficient $\kappa(t)$ is defined as

$$
\kappa(t)=\frac{\left\langle j_{+} \theta[r(+t)]\right\rangle_{R}-\left\langle j_{+} \theta[r(-t)]\right\rangle_{R}}{\left\langle j_{+}\right\rangle_{R}}
$$

and $\kappa$ is obtained from the plateau value of this function. In this expression, $j_{+}$represents the initially positive flux at $t=0$ which is simply given by $\dot{r}(t=0)$, where $r(t)$ is the $\mathrm{BrBr}$ separation as a function of time. $\theta(r)$ is a step function equal to one on the product side and zero on the reactant side. The averages are taken over the equilibrium distribution, reactive and non-reactive trajectories are therefore taken into account. The results obtained for the transmission coefficient are represented in Fig. 15. The decrease of $\kappa(t)$ is not as fast as in the $S_{N} 2$ reaction $^{4}$ due to the existence of recrossings at larger times in the present reaction. The asymptotic value is $0.44 \pm 0.05$ which can be compared to those obtained with classical MD simulations for the $S_{N} 1$ reaction $(0.53),{ }^{3}$
$S_{N} 2$ reaction $(0.55)^{4}$ and ion pair association $(0.15-0.18){ }^{2}$ In particular, the agreement between our value and that reported for the $S_{N} 1$ reaction, which is a process quite similar to ours, is quite satisfactory. The analysis of our results have shown that a reasonable value for the transmission coefficient is already obtained using the first 40 trajectories. Indeed, a plot of the transmission coefficient as a function of $n$, the number of trajectories used to compute it, displays strong variations below $n=40$ trajectories but oscillates only slightly between $n=40$ and $n=140$.

One may wonder what is the error introduced by the fact that we have considered a $\mathrm{BrBr}$ distance for the TS obtained from a SCRF calculation. A related question to this one is how the transmission coefficient would change by modifying the point on the reaction coordinate at which the flux from reactants to products is probed. Although it is not very easy to give conclusive answers to these questions, one must consider the fact that many recrossings have been predicted for large times. Hence, a small change of the $\mathrm{BrBr}$ distance at the TS and assuming unchanged trajectories, the curve $\kappa(t)$ would present slight changes for small times but would be essentially the same for large times.

\section{CONCLUSIONS}

This work is the first attempt to compute reaction trajectories in solution and dynamic solvent effects combining $a b$ initio (DFT in our case) calculations and rare event molecular dynamics simulations. This approach is quite computing demanding but provides a remarkably useful tool for analysing the role of the solvent on chemical reactions. Since the chemical system is described from first principles, there is no need to define an approximate potential, as happens in classical simulations. Compared to ab initio molecular dynamics, our approach is less computing demanding and renders possible a high level description of the chemical system through the use of basis set of any required quality. Besides, the hybrid DFT/MM approach allows one to study the fluctuations of the electronic properties such as the net atomic charges or the bond orders along the trajectories. This represent one of the most interesting features of the model which represents a choice method to carry out computer experiments of reactions in solution.

The application made in this paper concerns the first step of the electrophilic bromination of $\mathrm{CC}$ double bonds, which is a charge separation process in liquid water. Dynamic solvent effects have been found to be quite important as shown by the computed transmission coefficient $(0.44 \pm 0.05)$ which corresponds to a substantial departure from transition state theory. Barrier recrossings are obtained quite frequently even at large times. Besides, there are reactive trajectories in which cage effects are clearly apparent. This seems to be related to the existence of an energy well at the TS, a typical non-equilibrium effect. In general, the activation to the transition state takes about $200 \mathrm{fs}$ although some trajectories take longer. Substantial differences are found with respect to other studied systems through MD with classical potentials.

In the present paper, we have devoted our attention to 
the analysis of the dynamic solvent effects and the calculation of the transmission coefficient. In order to compute a rate constant, the activation free energy must be computed as well. This is also an expensive task and has not been made here but the computation does not present any particular difficulty and will be considered in future applications of the method.

\section{ACKNOWLEDGMENTS}

This work has been done within the C.N.R.S. Project G.D.R. No. 1017. M.S. is indebted to this project for providing him with a post-doctoral position at the Laboratoire de Chimie théorique de Nancy. The computations have been carried out on the IBM SP2 computer of the Centre National Universitaire Sud de Calcul (CNUSC) at Montpellier, France, (Project lct2550). We thank this institution for the computational facilities and for the help provided by its staff.

${ }^{1}$ R. M. Whitnell and K. R. Wilson, in Reviews in Computational Chemistry, edited by K. B. Lipkowitz, and D. B. Boyd (VCH, New York, 1993), p. $67-148$.

${ }^{2}$ M. Berkowitz, O. A. Karim, and P. J. Rossky, Chem. Phys. Lett. 105, 577 (1984); O. A. Karim and J. A. McCammon, J. Am. Chem. Soc. 108, 1762 (1986); Chem. Phys. Lett. 132, 219 (1986); G. Ciccotti, M. Ferrario, J. T. Hynes, and R. Kapral, J. Chem. Phys. 85, 925 (1988); Chem. Phys. 129, 241 (1989); J. Chem. Phys. 93, 7137 (1990).

${ }^{3}$ W. P. Keirstead, K. R. Wilson, and J. T. Hynes, J. Chem. Phys. 95, 5256 (1991)

4 (a) J. P. Bergsma, B. J. Gertner, K. R. Wilson, and J. T. Hynes, J. Chem. Phys. 86, 1356 (1987); (b) J. K. Hwang, G. King, S. Creighton, and A. Warshel, J. Am. Chem. Soc. 110, 5297 (1988); (c) B. J. Gertner, K. R. Wilson, and J. T. Hynes, J. Chem. Phys. 90, 3537 (1989); (d) B. J. Gertner, R. M. Whitnell, K. R. Wilson, and J. T. Hynes, J. Am. Chem. Soc. 113, 74 (1991)

${ }^{5}$ For a review, see, for instance, M. A. Fox, Chem. Rev. 92, 365 (1992).

${ }^{6}$ D. Borgis, S. Lee, and J. T. Hynes, Chem. Phys. Lett. 162, 19 (1989); A. Warshel and Z. T. Chu, J. Chem. Phys. 93, 4003 (1990); M. Nagaoka, Y. Okuno, and T. Yamabe, J. Am Chem. Soc. 113, 769 (1991); D. Borgis and J. T. Hynes, J. Chem. Phys. 94, 3619 (1991); J. J. I. Timoneda and J. T. Hynes, J. Phys Chem. 95, 10431 (1991); D. Borgis, G. Tarjus, and H. Azzouz, ibid. 96, 3188 (1992); J. Chem. Phys. 97, 1390 (1992); R. P. Muller and A. Warshel, J. Phys. Chem. 99, 17516 (1995); see also Ref. 10.

${ }^{7}$ For recent reviews, see J. Tomasi and M. Persico, Chem. Rev. 94, 2027 (1994); J. L. Rivail and D. Rinaldi, in Computational Chemistry, Reviews of Current Trends, edited by J. Leszczynski (World Scientific, Singapore, 1996), Vol. 1, pp. 139-174.

${ }^{8}$ M. A. Aguilar, F. J. Olivares del Valle, and J. Tomasi, J. Chem. Phys. 98, 7375 (1993); M. Cozzi, M. Persico, and J. Tomasi, J. Mol. Liq. 60, 87 (1994); M. F. Ruiz-López, D. Rinaldi and J. Bertrán, J. Chem. Phys. 103, 9249 (1995)

${ }^{9} \mathrm{~J}$. Gao, in Reviews in Computational Chemistry, edited by K. B. Lipkowitz and D. B. Boyd (VCH, New York, 1996), Vol. 7, pp. 119-185.

${ }^{10}$ I. Tuñón, M. T. C. Martins-Costa, C. Millot, and M. F. Ruiz-López, J. Chem. Phys. 106, 3633 (1997), preceding paper.

${ }^{11}$ I. Tuñón, M. T. C. Martins-Costa, C. Millot, and M. F. Ruiz-López, J. Mol. Mod. 1, 196 (1995).

${ }^{12}$ I. Tuñón, M. T. C. Martins-Costa, C. Millot, M. F. Ruiz-López, and J. L. Rivail, J. Comput. Chem. 17, 19 (1996).

${ }^{13}$ J. C. Keck, Faraday Discuss. Chem. Soc. 33, 173 (1962); J. B. Anderson, J. Chem. Phys. 58, 4684 (1973).

${ }^{14}$ J. B. Anderson, Adv. Chem. Phys. 91, 381 (1995).

${ }^{15}$ See Ref. 4(c) and references cited therein for a description on calculation of $\kappa$ in molecular dynamics simulations. See also Ref. 14 for a discussion on the differences between conversion and transmission coefficients.

${ }^{16}$ H. A. Kramers, Physica 7, 284 (1940).

${ }^{17}$ P. D. Bartlett and D. S. Tarbell, J. Am. Chem. Soc. 58, 466 (1936).
${ }^{18}$ J. E. Dubois and F. Garnier, Chem. Commun. 241 (1968); Bull. Soc. Chim. Fr. 3797 (1968); M. F. Ruasse and J. E. Dubois, J. Am. Chem. Soc. 97, 1977 (1975); C. Reichardt, Solvent Effect in Organic Chemistry (Verlag Chemie, New York, 1979), Chap. 5; M. F. Ruasse and S. Montallebi, J. Phys. Org. Chem. 4, 527 (1991).

${ }^{19}$ J. E. Dubois, F. Garnier, and H. Viellard, Tetrahedron Lett. 17, 1227 (1965); J. E. Dubois and F. Garnier, ibid. 26, 3047 (1966); G. A. Olah and T. R. Hockswender, Jr., J. Am. Chem. Soc. 96, 3574 (1974).

${ }^{20}$ M. F. Ruasse, Adv. Phys. Org. Chem. 28, 207 (1993).

${ }^{21}$ A. C. Hopkinson, M. H. Lien, K. Yates, and I. G. Czismadia, Theoret. Chim. Acta 38, 21 (1975); 44, 385 (1977).

${ }^{22}$ T. P. Hamilton and H. F. Shaefer III, J. Am. Chem. Soc. 112, 8260 (1990).

${ }^{23}$ S. Yamabe, T. Minato, and S. Inagaki, J. Chem. Soc., Chem. Commun. 532 (1988).

${ }^{24}$ T. Iwaoka, C. Kaneko, A. Shigihara, and H. Ichiwaka, J. Phys. Org. Chem. 6, 195 (1993).

${ }^{25}$ M. Jaszunski and E. Kochanski, J. Am. Chem. Soc. 99, 4624 (1977); E. Kochanski, in Quantum Theory of Chemical Reactions, edited by R. Daudel, A. Pullman, L. Salem, and A. Veillard (Reidel, Dordtrecht, 1980), Vol. 2, pp. 177-191.

${ }^{26}$ M. Cossi, M. Persico, and J. Tomasi, J. Am. Chem. Soc. 116, 5373 (1994).

${ }^{27}$ X. Assfeld, J. Garapon, D. Rinaldi, M. F. Ruiz-López, and J. L. Rivail, Theochem 371, 107 (1996)

${ }^{28}$ W. L. Jorgensen, J. Chandrashekar, J. D. Madura, R. W. Impey, and M. L. Klein, J. Chem. Phys. 79, 926 (1983)

${ }^{29}$ M. T. C. Martins-Costa, B. Finance, C. Millot, and M. F. Ruiz-López (work in progress).

${ }^{30}$ J. L. Rivail, D. Rinaldi, and M. F. Ruiz-López, in Theoretical and Computational Models for Organic Chemistry, NATO ASI Series C, Vol. 339, edited by S. J. Formosinho, I. G. Csizmadia, and L. Arnaut (Kluwer Academic, Dordrecht, 1991), pp. 79-92; D. Rinaldi, J. L. Rivail, and N. Rguini, J. Comput. Chem. 13, 675 (1992); V. Dillet, D. Rinaldi, J. G. Angyán, and J. L. Rivail, Chem. Phys. Lett. 202, 18 (1993); M. F. RuizLópez, F. Bohr, M. T. C. Martins-Costa, and D. Rinaldi, ibid. 221, 109 (1994).

${ }^{31}$ X. Assfeld, J. González, R. López, M. F. Ruiz-López, J. A. Sordo, and T. L. Sordo, J. Comput. Chem. 15, 479 (1994); M. F. Ruiz-López, X. Assfeld, J. I. García, J. A. Mayoral, and L. Salvatella, J. Am. Chem. Soc. 115, 8780 (1993); S. Antonczak, M. F. Ruiz-López, and J. L. Rivail, ibid. 116, 3912 (1994); I. Tunón, D. Rinaldi, M. F. Ruiz-López, and J. L. Rivail, J. Phys. Chem. 99, 3798 (1995)

${ }^{32}$ R. López, M. F. Ruiz-López, D. Rinaldi, J. A. Sordo, and T. L. Sordo, J. Phys. Chem. 100, 10600 (1996).

${ }^{33}$ S. W. de Leeuw, J. W. Perram, and H. G. Petersen, J. Stat. Phys. 61, 1203 (1990).

${ }^{34}$ M. P. Allen and D. J. Tildesley, Computer Simulation of Liquids (Clarendon, Oxford, 1989).

${ }^{35}$ S. Nosé, Mol. Phys. 52, 255 (1984); W. G. Hoover, Phys. Rev. A 31, 1695 (1985); S. Nosé, Mol. Phys. 57, 187 (1986).

${ }^{36}$ H. C. Andersen, J. Chem. Phys. 72, 2384 (1980).

${ }^{37}$ M. Strnad, C. Millot, M. T. C. Martins-Costa, and M. F. Ruiz-López (work in progress).

${ }^{38}$ L. D. Landau and E. M. Lifshitz, Statistical Physics (Pergamon, London, 1958).

${ }^{39}$ I. Benjamin, B. J. Gertner, N. J. Tang, and K. R. Wilson, J. Am. Chem. Soc. 112, 524 (1990).

${ }^{40}$ M. E. Tuckerman, B. J. Berne, and G. J. Martyna, J. Chem. Phys. 97, 1990 (1992); A. Rossi, ibid. 94, 1465 (1990); G. J. Martyna and B. J. Berne, ibid. 94, 6811 (1991); M. E. Tuckerman and B. J. Berne, ibid. 95, 8362 (1991); J. Phys. Chem. 98, 6885 (1994).

${ }^{41}$ S. H. Vosko, L. Wilk, and M. Nusair, Can. J. Phys. 58, 1200 (1980).

${ }^{42}$ A. St-Amant and D. Salahub, Chem. Phys. Lett. 169, 387 (1990); D. Salahub, R. Fournier, P. Mlynarski, I. Papai, A. St-Amant, and J. Ushio, in Theory and Applications of Density Functional Approaches to Chemistry, edited by J. Labanowski and J. Andzelm (Springer, Berlin, 1991).

${ }^{43}$ A. D. Becke, Phys. Rev. A 38, 3098 (1988); J. P. Perdew, Phys. Rev. B 33, 8822 (1986); 34, 7406 (E) (1986).

${ }^{44}$ (a) T. P. Lybrand, I. Ghosh, and J. A. McCammon, J. Am. Chem. Soc. 107, 7793 (1985); (b) W. L. Jorgensen, in Biochemical and Organic Simulation System (BOSS), Version 3.5 (Yale University, New Haven, 1994).

${ }^{45}$ D. Fincham, CCP5 Q. 2, 6 (1981); D. Fincham and D. M. Heyes, Adv. Chem. Phys. 68, 493 (1985). 
${ }^{46}$ L. Verlet, Phys. Rev. 159, 98 (1967); W. C. Swope, H. C. Andersen, P. H. Berens, and K. R. Wilson, J. Chem. Phys. 76, 637 (1982).

${ }^{47}$ J. P. Ryckaert, G. Ciccotti, and H. J. C. Berendsen, J. Comput. Phys. 23, 327 (1977).

${ }^{48}$ Constraining the reaction coordinate during the NVT equilibration of the transition state introduces both a configurational and zero velocity constraint. Average over the constrained ensemble is not rigorous and may be affected by some error. For semirigid systems, this error may be avoided using the approach of Ryckaert et al. [J. P. Ryckaert and G. Ciccotti, Mol. Phys. 58, 1125 (1986)] but this is not the case for flexible molecules. In our case, we use SHAKE to constrain the $\mathrm{BrBr}$ distance so that the corresponding relative velocity is unconstrained. In other words, the reaction coordinate is restricted only [E. A. Carter, G. Ciccotti, J. T. Hynes, and R. Kapral, Chem. Phys. Lett. 156, 472 (1989)].

${ }^{49}$ E. Ruiz, D. R. Salahub and A. Vela, J. Am. Chem. Soc. 117, 1141 (1995).

${ }^{50}$ The location of the transition state has been made using GAUSSIAN 92/DFT (Ref. 51) using different exchange-correlation functionals and basis sets. All these calculations showed close structures for the transition state and in particular the reaction coordinate was predicted to be essentially defined by the $\mathrm{BrBr}$ bond length in all cases. Using these results, the transition state structure in Table I has been obtained using deMon (Ref. 42) assuming that the reaction coordinate near the transition state is the $\mathrm{BrBr}$ bond length.

${ }^{51}$ M. J. Frisch, G. W. Trucks, H. B. Schlegel, P. M. W. Gill, B. G. Johnson, M. W. Wong, J. B. Foresman, M. A. Robb, M. Head-Gordon, E. S. Replogle, R. Gomperts, J. L. Andrés, K. Raghavachari, J. S. Binkley, C. Gonzalez, R. L. Martin, D. J. Fox, D. J. Defrees, J. Baker, J. J. P. Stewart, and J. A. Pople, Gaussian 92/DFt, Revision G.1, Gaussian, Inc., Pittsburgh, 1993.

${ }^{52}$ R. Car and M. Parrinello, Phys. Rev. Lett. 55, 2471 (1985).

${ }^{53}$ K. Laasonen, M. Sprik, and M. Parrinello, J. Chem. Phys. 99, 9080 (1993).

${ }^{54}$ I. Tuñón, M. T. C. Martins-Costa, C. Millot, and M. F. Ruiz-López, Chem. Phys. Lett. 241, 450 (1995).

${ }^{55} \mathrm{P}$. А․ Bergström, J. Lindgren, and O. Kristiansson, J. Phys. Chem. 95, 8575 (1991)

${ }^{56}$ W. L. Jorgensen, J. K. Buckner, S. E. Huston, and P. J. Rossky, J. Am. Chem. Soc. 109, 1891 (1987).

${ }^{57}$ J. P. Bergsma, J. R. Reimers, K. R. Wilson, and J. T. Hynes, J. Chem. Phys. 85, 5625 (1986); see also Refs. 16, 53-54 cited in Ref. 4(a). 